\title{
Segregierte Schulmilieus, variierende Unterrichtsbedingungen und Lernleistungen der Schülerinnen und Schüler. Eine Analyse mit Daten der Grundschulen in Rheinland-Pfalz 2015/16
}

\author{
Thorben Kraus • Horst Weishaupt • Ingmar Hosenfeld
}

Eingegangen: 6. Oktober 2020 / Angenommen: 4. November 2020 / Online publiziert: 12. Januar 2021

(C) Der/die Autor(en) 2021

Zusammenfassung Auf der Grundlage der VERA3-Daten der Grundschulen in Rheinland-Pfalz wird der Zusammenhang zwischen der sozialen Zusammensetzung der Schülerinnen und Schüler der einzelnen Schulen und den durchschnittlichen Leistungen im Leseverstehen und in Mathematik analysiert. Besonders interessiert der vermittelnde Einfluss der schulstatistisch erfassten schul- und unterrichtsorganisatorischen Bedingungen, weil sie - im Gegensatz zu der familiären Situation der Schülerinnen und Schüler - schulpolitisch beeinflusst werden können. Neben deskriptiven Befunden wird ein Pfadmodell berechnet, in das die schulorganisatorischen Variablen als vermittelnde Prozessvariablen des Zusammenhangs zwischen Merkmalen der Schülerschaft und den gemessenen durchschnittlichen Leistungen enthalten sind.

Schlüsselwörter VERA3 - Schülerleistungen · Schulorganisatorische Bedingungen $\cdot$ Schulstatistik $\cdot$ Nichtdeutsche Familiensprache $\cdot$ Lernmittelfreiheit

T. Kraus, M.A.

Zentrum für Empirische Pädagogische Forschung, Universität Koblenz-Landau,

Bürgerstr. 23, 76829 Landau i.d. Pfalz, Deutschland

E-Mail: Kraus@zepf.uni-landau.de

Prof. i. R. H. Weishaupt $(\square)$

DIPF | Leibniz-Institut für Bildungsforschung und Bildungsinformation,

Rostockerstr. 29, 60323 Frankfurt am Main, Deutschland

E-Mail: weishaupt@dipf.de

Prof. I. Hosenfeld

Zentrum für Empirische Pädagogische Forschung, Pädagogisch-psychologische Bildungsforschung, Universität Koblenz-Landau, Bürgerstr. 23, 76829 Landau i.d. Pfalz, Deutschland

E-Mail: hosenfeld@zepf.uni-landau.de 


\title{
School segregation, varying instructional organization of schools and achievment of pupils. A study with primary school data of Rhineland- Palatinate
}

\begin{abstract}
Drawing on data from the VERA3 study of primary schools in RhinelandPalatinate, the authors analyze the correlation between social compositions of students in individual schools and average reading and mathematics achievement. Particular attention is paid to the mediating influence of school statistically relevant conditions of school and instructional organization, which-other than the family background — can be influenced by educational policy-making. Alongside presenting descriptive findings, the authors calculate a path model which contains the school organizational variables as mediating process variables of the correlation between student population variables and measured average achievement.
\end{abstract}

Keywords VERA3 - Student achievement - School organizational conditions · School statistics $\cdot$ Non-German language spoken at home $\cdot$ Free learning material

\section{Einleitung und Forschungsfragen}

Die räumliche Konzentration sozialer Gruppen gewinnt in den letzten Jahren in den Bemühungen um die Verringerung sozialer Benachteiligung im Schulwesen wachsende Aufmerksamkeit. Die sich verschärfenden Probleme am Wohnungsmarkt in vielen Großstädten markieren nur die jüngste Zuspitzung eines langfristigen Prozesses zunehmender Segregation und damit auch sozialer Entmischung, die sich im Schulwesen noch verstärkt zeigt. Aus der Stadtsoziologie sind die sozialen Disparitäten innerhalb der Städte und fortschreitende Prozesse der sozialen Differenzierung bekannt und in ihren Auswirkungen auf Lebensbedingungen und -chancen unstrittig (s. zusammenfassend: Sundsboe 2015). Neben innerstädtischen Disparitäten sind auch Stadt-Land-Unterschiede zu beachten (Beierle et al. 2019), die vor allem durch unterschiedliche Lebensstile gekennzeichnet werden können, durch Unterschiede in den vorherrschenden Familien-, Haushalts- und Wohnformen (Eigentum/Miete, EinMehrfamilienhaus). Außerdem gibt es eine große Stabilität in den sozialen Unterschieden an den Schulen, wie beispielsweise eine Langzeitstudie zum Ruhrgebiet eindrucksvoll zeigen konnte (Jeworutzki et al. 2017). Überblicksanalysen verdeutlichen, dass es sich dabei um keine Randerscheinung des Schulsystems handelt, sondern schätzungsweise bundesweit ein Viertel der Schülerinnen und Schüler an Grundschulen in relativ stark segregierten schulischen Milieus mit einem hohem Anteil von SGBII-Empfängern und/oder Migranten unterrichtet werden (Weishaupt 2016, S. 359). Zahlreiche Forschungsbefunde verweisen inzwischen darauf, dass die Segregation zwischen den Grundschulen größer ist als die Wohnsegregation (s. beispielsweise: Morris-Lange et al. 2013, S. 7 ff.). Dafür sind die zunehmende Zahl von Privatschulen, die sozial segregierend wirken (Klemm et al. 2018, S. 38-41), und die Möglichkeiten der Umgehung des Besuchs der zuständigen Schule über Gestattungen und die Aufhebung von Schulbezirken verantwortlich (Finke und Lange 2012). In den Städten hat ein hoher Anteil von privaten Grundschulen aber eine ab- 
schwächende Auswirkung auf die Wohnsegregation (Helbig und Jähnen 2018). Die vorliegenden Forschungsbefunde legen es nahe, sozialräumliche Unterschiede über Schülermerkmale der jeweiligen Schule zu erfassen und nicht über Merkmale des Bezirks, in dem die Schule gelegen ist (Morris-Lange et al. 2013, S. 17; Schuchart et al. 2012).

Die Konzentration von Schülerinnen und Schülern unterer Sozialgruppen an Schulen führt - so zeigt vor allem die internationale Forschung - häufig noch zu einer Verstärkung sozial benachteiligender Faktoren (Ditton und Krüsken 2006; Dumont et al. 2013). Dazu tragen vermutlich neben Merkmalen sozialen Verhaltens (geringe Leistungserwartungen an die Schülerinnen und Schüler, nicht an die schulische Situation angepasste Schulkultur etc., Bremm 2020) sozial benachteiligende schulorganisatorische und personelle Bedingungen bei.

Bezogen auf die Grundschule mehren sich die Forschungsbefunde, die Zweifel daran aufkommen lassen, dass die Schulverwaltung konsequent wenigstens auf gleiche schulorganisatorische und personelle Bedingungen an allen Schulen achtet. In den Ländern Hessen und Nordrhein-Westfalen sollen „,sozial belastete Schulen“ besser personell ausgestattet werden. Doch lassen sich keine oder sogar negative Effekte einer belastenden sozialen Situation auf die Personalausstattung der Grundschulen nachweisen (Weishaupt und Kemper 2016; Weishaupt 2018a). Schon vor mehreren Jahren gab es durch Presseartikel Hinweise darauf, dass „Schulen in einem sozioökonomisch benachteiligten Umfeld erstens größere Schwierigkeiten haben, den Wettbewerb um qualifizierte Fachkräfte zu gewinnen und zweitens dauerhaft einen hohen Anteil von erkrankten Lehrkräften verbuchen“" (Sundsboe 2015, S. 57). Hinzu kommen eine oft größere Fluktuation unter den Lehrkräften und bei den Schulleitungsstellen (Bremm et al. 2016, S. 331). In einer neuen Studie konnte die Konzentration von Seiteneinsteigern unter den Lehrkräften in Schulen mit einer durchschnittlich niedrigen sozialen Lebenslage der Schülerinnen und Schüler nachgewiesen werden (Richter et al. 2018). Im Rahmen der IQB-Erhebungen wurde beobachtet, dass fachfremder Unterricht in Deutsch und Mathematik verstärkt an Grundschulen mit einem hohen Anteil von Schülerinnen und Schülern mit Migrationshintergrund oder einem niedrigen durchschnittlichen kognitiven Leistungsniveau vorkommt (Ziegler und Richter 2017, S. 149). Die Beispiele von Hessen und Nordrhein-Westfalen zeigen, dass intendierte Maßnahmen nicht umgesetzt wurden. In Berlin, wo durch Zusatzzuweisungen von Personal für Sprachförderung und für die Förderung von armen Kindern ebenfalls eine bessere Personalausstattung der Schulen mit dem Bedarf an Fördermaßnahmen zu erwarten ist, zeigt eine Auswertung der Daten der Schulstatistik: „Insgesamt kommen wir zu dem Befund, dass die sozial am stärksten benachteiligten Schulen auch die ungünstigsten Rahmenbedingungen aufweisen. An sozial benachteiligten Schulen ist die Personalabdeckung schlechter, Unterricht muss häufiger vertreten werden oder fällt aus und an ihnen arbeiten mehr Lehrkräfte ohne Lehramtsstudium als an Schulen mit einer besseren sozialen Zusammensetzung. Zudem sind an diesen Schulen die in den Schulinspektionen gemessenen Qualitätsbereiche schlechter" (Helbig und Nikolai 2019, S. 25-26).

Diese Befunde sind für die Verringerung sozialer Benachteiligungen deshalb von besonderem Interesse, weil Unterschiede in der Personalausstattung und den Rahmenbedingungen des Unterrichts der Schulen unmittelbar Folgen der schulpoliti- 
schen Steuerung sind und nicht, wie die soziale Lage und Herkunft der Familie der Schülerinnen und Schüler der schulpolitischen Steuerung entzogen. Bemerkenswert ist aber, dass in der Schulleistungsforschung in Deutschland der vermittelnde Einfluss schulorganisatorischer und personeller Bedingungen auf die Schülerleistungen kaum untersucht wurde, weil beispielsweise eine Verknüpfung der Daten der IQB-Erhebungen mit Daten der Schulstatistik zu den schulorganisatorischen Bedingungen und der Personalausstattung an den Schulen bislang ausgeschlossen ist ${ }^{1}$. Teilweise erschwert die Ziehung sehr kleiner Stichproben an den einzelnen Schulen entsprechende Analysen. Umso wichtiger erscheint es, Möglichkeiten zu nutzen, der Schulpolitik Hinweise zu geben, mit welchen schulpolitischen Steuerungsimpulsen sie den Zusammenhang von sozialer Lage und Schülerleistungen verringern kann.

Rheinland-Pfalz bietet für die Analyse dieses Anliegens eine günstige schulstatistische Datenbasis. Außerdem haben bisherige Studien $\mathrm{zu}$ den personellen und schulorganisatorischen Bedingungen an den Schulen in Rheinland Pfalz einige Hinweise auf Zusammenhänge zwischen den sozialen Bedingungen an den Schulen, dem Anteil der Inklusionsschülerinnen und -schülern und schulorganisatorischen und personellen Bedingungen an den Schulen geführt (Weishaupt 2018b, 2020). Außerdem konnte dort der Zugang zu den Daten der VERA3-Erhebung im Schuljahr 2015/16 erreicht werden ${ }^{2}$, und damit zu Leistungsdaten nahezu aller öffentlichen Grundschulen in Deutsch und Mathematik, um sie mit den schulstatistischen Daten für Analysen auf Schulebene zu verknüpfen. Die Zielrichtung der dadurch ermöglichten Analysen lässt sich anhand folgender Forschungsfragen erläutern:

1. Gibt es Leistungsunterschiede zwischen Schulen in Abhängigkeit von dem Anteil der Schülerinnen und Schüler mit nichtdeutscher Familiensprache/Befreiung von der Lernmittelbezahlung? Wie wirkt sich der Anteil von Schülerinnen und Schülern mit sonderpädagogischem Förderbedarf auf die durchschnittliche Leistung der Schülerinnen und Schüler ohne sonderpädagogischen Förderbedarf aus? Gibt es verstärkende Effekte, wenn zwei oder alle drei Faktoren zusammenkommen?

2. Wie groß sind die Leistungsunterschiede? Sind die Unterschiede zwischen dem Leseverstehen und Mathematik unterschiedlich stark?

3. Gibt es einen vermittelnden Einfluss der schulorganisatorischen Bedingungen und der Personalausstattung auf die durchschnittlichen Schülerleistungen an den Grundschulen?

Nachfolgend beschreiben wir zunächst die der Studie zugrunde liegenden schulstatistischen Daten und das Datenmaterial der VERA3-Erhebung. Anschließend erläutern wir die methodischen Schritte der Analysen. Nach der Präsentation der Ergebnisse ziehen wir ein Fazit.

\footnotetext{
1 So das Ergebnis der Recherchen beim FDZ des IQB, weil die offiziellen Schulnummern nach der Erhebung und vor der Weitergabe an das IQB entfernt werden. Es gibt auch keine Absprache über die Bereitstellung einzelschulischer schulorganisatorischer Daten der Schulstatistik (orientiert am Kerndatensatz der KMK), die den Daten vor der Weitergabe an das IQB zugespielt werden könnten.

2 Ermöglicht wurde diese Studie durch den Rechtsschutz der Gewerkschaft Erziehung Wissenschaft, der unser Dank gilt. Dadurch wurde der Zugang zu den VERA-Daten in Kooperation mit dem zepf ermöglicht.
} 


\section{Zum Datenmaterial der Studie}

Rheinland-Pfalz hat seine Schulstatistik frühzeitig entsprechend der Absprachen in der Kultusministerkonferenz auf eine Individualstatistik umgestellt (Mundelius 2019), orientiert an dem zwischen den Ländern vereinbarten Kerndatensatz. Vor allem sind diese Daten aber auch in vollem Umfang der Forschung zugänglich, während andere Länder den Zugang zu verfügbaren Daten der Schulstatistik verweigern, weil der Forschungszugang zur Schulstatistik als Behördenstatistik der Kultusministerien nach Landesrecht teilweise nicht geregelt ist (Hanschmann und Weishaupt 2017).

\subsection{Merkmale der Zusammensetzung der Schülerschaft}

Die Berücksichtigung der sozialen Lage der Schülerinnen und Schüler ist über die Schulstatistik nicht umstandslos möglich, weil sie keine Angaben zum Beruf, Einkommen und Bildungsniveau der Eltern vorsieht, den zentralen Indikatoren zur Bestimmung der sozialen Lage. Deshalb wird der Migrationshintergrund und speziell die nichtdeutsche Familiensprache als Ersatzmerkmal herangezogen. Dabei ist zu beachten, dass Migrantinnen und Migranten sich in Deutschland im Vergleich zu Nichtmigrantinnen und Nichtmigranten im Durchschnitt in einer deutlich niedrigeren sozialen Position befinden, die auch in einem niedrigeren Bildungsstand und häufigerer Armut zum Ausdruck kommt (Autorengruppe Bildungsberichterstattung 2016, S. 168-169). Doch sind die Angaben zum Migrationshintergrund kein wirklich befriedigender Ersatzindikator. Er kennzeichnet nicht nur einen durchschnittlich niedrigeren sozialen Status der Schülerinnen und Schüler, sondern auch kulturelle Unterschiede. Hinzu kommt, dass die Schulstatistik keine Merkmale der Eltern der Schülerinnen und Schüler erhebt, wodurch sich häufig Untererfassungen gegenüber der Einwohnerstatistik ergeben (Kemper 2017). Im Schuljahr 2015/16 hatten nach der Schulstatistik in Rheinland-Pfalz 25,5\% der Grundschülerinnen und Grundschüler einen Migrationshintergrund, nach dem Mikrozensus von 2015 aber 36,3\% der Kinder zwischen 6 und 9 Jahren (Weishaupt 2018b, S. 260) und nach der IQB-Erhebung 37,2\% der befragten Viertklässlerinnen und Viertklässler (Rjosk et al. 2017, S. 242), nur etwa $70 \%$ der Schülerinnen und Schüler mit Migrationshintergrund werden folglich von der Schulstatistik erfasst ${ }^{3} .24,4 \%$ der Grundschülerinnen und Grundschüler hatten eine nichtdeutsche Familiensprache ${ }^{4}$. Da mit diesem Indikator

\footnotetext{
3 Nach der Bevölkerungsstatistik und der IQB-Erhebung hat ein Kind einen Migrationshintergrund, wenn wenigstens ein Elternteil im Ausland geboren ist oder eine nichtdeutsche Staatsbürgerschaft hat. Nach der Schulstatistik muss die Schülerin/der Schüler eines der folgenden drei Merkmale aufweisen: im Ausland geboren, keine deutsche Staatsbürgerschaft, eine nichtdeutsche Familiensprache.

${ }^{4}$ Die Tatsache, dass $96 \%$ der von der Schulstatistik erfassten Grundschülerinnen und Grundschüler mit Migrationshintergrund eine nichtdeutsche Familiensprache haben, lässt an der Validität der Angaben zum Migrationshintergrund zweifeln. Bei den ausländischen Schülern stimmen die Angaben zwischen Mikrozensus und Schulstatistik zu $92 \%$ überein (8,4 zu 7,7\%). Die Kindertagesstättenstatistik erfasst, ob wenigstens ein Elternteil aus dem Ausland zugewandert ist. Von den 3 bis unter 6-jährigen Kindern in Kindertageseinrichtungen in Rheinland-Pfalz hatten 2015 34,1\% mindestens ein zugewandertes Elternteil (Weishaupt 2018b).
} 
auch ein durchschnittlich höherer Sprachförderbedarf verbunden ist, wird er hier als erstes Merkmal der sozialen Lage der Schülerinnen und Schüler verwendet.

Als Indikator für eine niedrige soziale Lage kann in Rheinland-Pfalz zweitens der Anteil der Schülerinnen und Schüler herangezogen werden, denen nach der ,Landesverordnung über die Lernmittelfreiheit und die entgeltliche Ausleihe von Lernmitteln' bei Unterschreitung bestimmter Einkommensgrenzen die Lernmittel auf Antrag kostenlos zur Verfügung gestellt werden. Die darüber im Kultusministerium geführte Statistik nach einzelnen Schulen wurde auf Anfrage zur Verfügung gestellt. Der Anteil der Empfänger dieser Leistungen unter den Grundschülerinnen und -schülern ist im Schuljahr 2015/16 mit 25,6\% etwa doppelt so hoch wie der Anteil der Empfänger von Hilfen zum Lebensunterhalt von 6 bis 9 Jahren in Rheinland-Pfalz von 12,7\% (Dezember 2015). Der Vorteil dieses Indikators ist der direkte Bezug zu den Schülerinnen und Schülern einzelner Schulen, während die Daten zu den Leistungsempfängern nach dem SGB II nur für Gemeinden und Stadtteile, in denen Schulen gelegen sind, zur Verfügung stehen.

Die beiden Indikatoren zur nichtdeutschen Familiensprache und dem Anteil der Schülerinnen und Schüler mit Lernmittelfreiheit sollen die sozialen Bedingungen an den einzelnen Schulen erfassen. Zwischen beiden Indikatoren besteht auf der Basis der analysierten öffentlichen Grundschulen eine Korrelation von $r=0,643$, also ein sehr hoher Zusammenhang.

Mit dem Anteil der Schülerinnen und Schüler mit sonderpädagogischem Förderbedarf ${ }^{5}$ wird ein weiteres Merkmal der Zusammensetzung der Schülerinnen und Schüler der einzelnen Schulen berücksichtigt, das spezifische pädagogische Bedingungen der Unterrichtsorganisation erfasst. Drei Viertel der Inklusionsschülerinnen und -schüler an Grundschulen in Rheinland-Pfalz haben den Förderschwerpunkt Lernen. Unter diesen Schülerinnen und Schülern sind Kinder unterer Sozialgruppen überrepräsentiert. Folglich besteht ein positiver Zusammenhang zwischen dem Anteil von Inklusionsschülerinnen und -schülern und dem Anteil von Schülerinnen und Schülern mit Lernmittelbefreiung $(r=0,227)$ und nichtdeutscher Familiensprache $(r=0,168)$ an den einzelnen Grundschulen. Die Schulen mit einer durchschnittlich niedrigen sozialen Lage der Schülerinnen und Schüler stehen dadurch überzufällig häufig durch die ebenfalls zu unterrichtenden Inklusionsschülerinnen und -schüler vor vielfältigen und nicht selten divergenten pädagogischen Herausforderungen.

Den Merkmalen der Zusammensetzung der Schülerschaft werden durch die Schulstatistik verfügbare Informationen $\mathrm{zu}$ den schulorganisatorischen und personellen Bedingungen an den einzelnen Schulen gegenübergestellt. Die Kultusministerkonferenz berichtet regelmäßig in ihren Statistiken zur Schulentwicklung die Zahl der Schüler je Klasse und die Zahl der wöchentlich erteilten Unterrichtsstunden je Schüler und je Klasse (Sekretariat der KMK 2020). Zur Klassengröße gibt es zahlreiche und im Ergebnis nicht einheitliche Forschungsbefunde (s. Avenarius et al. 2003, S. 110-115). Bezogen auf die erteilten Unterrichtsstunden werden hier die Unterrichtsstunden je 100 Schülerinnen und Schüler verwendet, weil dieser

\footnotetext{
5 Bei Schulen mit nur einem oder zwei Inklusionsschülerinnen und -schülern wurde die Häufigkeit bei der Lieferung der Daten aus Datenschutzgründen unterdrückt und durch den Wert 1,51 ersetzt, der zur Summe der statistisch ausgewiesenen Grundschüler an Inklusionsschulen führt.
} 
Indikator nicht durch unterschiedliche Klassenfrequenzen beeinflusst wird und damit unabhängig von einzelschulischen Entscheidungen zum Personaleinsatz deren Verfügbarkeit je Schüler anzeigt. Zusätzlich steht der Anteil des von nicht vollqualifizierten Lehrkräften ${ }^{6}$ erteilten Unterrichts an den Wochenunterrichtsstunden zur Verfügung.

\subsection{Merkmale der Schulorganisation}

Zusätzlich über die Schulstatistik verfügbare schulorganisatorische Merkmale sind die Schulgröße (erfasst über die Schülerzahl), Ganztagsangebote an der Schule (ja/ nein), die Angabe, ob es sich um eine Schwerpunktschule zur sonderpädagogischen Förderung (ja/nein) handelt und ob es an der Schule jahrgangsübergreifende Klassen gibt (ja/nein).

Die schulorganisatorischen Merkmale sind teilweise untereinander hoch korreliert. Deshalb werden die Schulgröße und die Bezeichnung als Schwerpunktschule im Folgenden nicht mehr berücksichtigt, denn die durchschnittliche Klassengröße korreliert stark mit der Schülerzahl der Schulen $(r=0,711)$ und ebenso das Merkmal Schwerpunktschule mit dem Anteil der Schülerinnen und Schüler mit sonderpädagogischem Förderbedarf $(r=0,813)$.

Zwischen den Merkmalen zur Zusammensetzung der Schülerinnen und Schüler der Schulen und den schulorganisatorischen und personellen Bedingungen bestehen Zusammenhänge, die es sinnvoll erscheinen lassen, sie in ihren Einflüssen auf die Schülerleistung weiter zu untersuchen. Meist stimmen die Zusammenhänge zwischen Merkmalen der Zusammensetzung der Schülerinnen und Schüler an den Schulen und den schulorganisatorischen Bedingungen überein (s. Tab. 1): mit der Zunahme des Anteil der Schülerinnen und Schüler mit nichtdeutscher Familiensprache, mit Lernmittelfreiheit oder dem Anteil der Schülerinnen und Schüler mit sonder-

Tab. 1 Zusammenhänge zwischen Merkmalen der Zusammensetzung der Schülerschaft und schulorganisatorischen Bedingungen an den Grundschulen in Rheinland-Pfalz 2015/16 ( $N=872)$

\begin{tabular}{|c|c|c|c|}
\hline \multirow[b]{2}{*}{ Schulorganisatorische Merkmale } & \multicolumn{3}{|c|}{ Anteil der Schülerinnen und Schüler mit ... } \\
\hline & $\begin{array}{l}\text { Lernmittel- } \\
\text { freiheit }\end{array}$ & $\begin{array}{l}\text { Nichtdeutscher } \\
\text { Familiensprache }\end{array}$ & $\begin{array}{l}\text { Sonderpädagogischem } \\
\text { Förderbedarf }\end{array}$ \\
\hline Schulen mit Ganztagsangeboten & $0,268 * *$ & $0,261 * *$ & $0,183 * *$ \\
\hline $\begin{array}{l}\text { Wöchentlich erteilte Unterrichts- } \\
\text { stunden je } 100 \text { Schüler*innen }\end{array}$ & $0,104 * *$ & $-0,096 * *$ & $0,472 * *$ \\
\hline Schüler*innen je Klasse & $0,260 * *$ & $0,410 * *$ & $0,187 * *$ \\
\hline Jahrgangsübergreifende Klassen & $-0,139 * *$ & $-0,235^{* *}$ & $-0,084$ \\
\hline $\begin{array}{l}\text { Von nicht voll qualifizierten Lehr- } \\
\text { kräften erteilter Wochenstunden- } \\
\text { anteil }\end{array}$ & 0,032 & 0,016 & $0,060 *$ \\
\hline
\end{tabular}

$* p<0,05 ; * * p<0,01$

\footnotetext{
6 Dabei handelt es sich um Lehrkräfte ohne Lehramtsstudium und ohne Vorbereitungsdienst und sonstige Lehramtsprüfung (z. B. im Ausland erworbene), Pädagogische Fachkräfte mit Zusatzbefähigung in Sonderpädagogik, Lehramtsanwärter/Quer-/Seiteneinsteiger/-in mit angestrebtem Abschluss LA an Grund- und Hauptschulen, Förderschulen oder Realschulen, Referendar/Quer-/Seiteneinsteiger/-in mit angestrebtem Abschluss Lehramt an Gymnasien oder berufsbildenden Schulen.
} 
pädagogischem Förderbedarf nimmt die Häufigkeit eines ganztägigen Angebots an den Schulen zu, es steigt aber auch die durchschnittliche Zahl der Schüler je Klasse. Grundschulen mit jahrgangsübergreifenden Klassen sind durchschnittlich günstiger sozial zusammengesetzt. Unterschiedlich ist die Situation bei den wöchentlich erteilten Unterrichtsstunden je 100 Schüler: An Schulen mit einem hohen Anteil von Schülerinnen und Schülern mit Lernmittelfreiheit ist auch die Lehrkräfteversorgung durchschnittlich besser, an Schulen mit einem hohen Anteil von Schülerinnen und Schülern mit nichtdeutscher Familiensprache eher schlechter. Ein Anstieg der erteilten Unterrichtsstunden ist mit der Zunahme des Anteils der Inklusionsschülerinnen und -schüler verbunden. Der Anteil des von nicht voll qualifizierten Lehrkräften erteilten Unterrichts variiert unabhängig von der sozialen Situation der Schülerinnen und Schüler an den Grundschulen und variiert nur leicht mit dem Anteil der Inklusionsschülerinnen und -schüler.

\subsection{Leistungsdaten aus Vergleichsarbeiten}

Die Vergleichsarbeiten sind eingebunden in die Gesamtstrategie zum Bildungsmonitoring der KMK $(2006,2016)$ als ein „Verfahren zur Qualitätssicherung auf Ebene der Schulen“ (KMK 2016, S. 13). Sie sollen den Schulen einerseits eine Standortbestimmung in Bezug auf die Bildungsstandards ermöglichen und andererseits helfen, die kompetenzbezogenen Konzepte der Bildungsstandards zu vermitteln. Sie stellen so einen Impuls für die innere Schulentwicklung dar. Dennoch werden die Erhebungsergebnisse auch für weitergehende Analysen (Laschke 2010) und für landesweite Auswertungen verwendet (z. B. Bayerisches Landesamt für Schule 2018, S. 89-93). Rheinland-Pfalz hat bisher auf landesweite Ergebnisdarstellungen verzichtet.

Die Vergleichsarbeiten in Jahrgangsstufe 3 werden in Rheinland-Pfalz verpflichtend für alle öffentlichen Grundschulen als Paper-Pencil-Test durchgeführt, wobei für alle Schülerinnen und Schüler des Landes identische Testhefte zum Einsatz kommen. Für das Schuljahr 2015/2016 stehen für alle Schulen Leistungsmaße für das Leseverständnis und Mathematik (mit den Leitideen Zahlen \& Operationen sowie Muster \& Strukturen) zur Verfügung. In die hier vorgelegten Analysen gingen alle öffentlichen Grundschulen ein, die einer Nutzung Ihrer Daten innerhalb einer Widerspruchsfrist zu Beginn des Schuljahres 2019/2020 nicht widersprochen haben. Insgesamt 39 Grundschulen (4,28\%) widersprachen der Verwendung ihrer Daten.

Für die Rückmeldungen der Vergleichsarbeiten werden die Schülerleistungen im Rahmen einer IRT-Skalierung anhand der vom IQB in Pilotierungsstudien ermittelten Itemparameterschätzungen bestimmt und auf der Ebene der Zuordnung zu den Kompetenzstufen der Bildungsstandards den Schulen mitgeteilt. Für die hier berichteten Analysen wird auf die rohen Personenparameterschätzer (d.h. vor der Transformation auf die Metrik der Bildungsstandards) zugegriffen. Diese wurden im ersten Arbeitsschritt auf Schulebene aggregiert, wobei für Mathematik die Parameter der beiden Inhaltsbereiche gemittelt wurden. Schülerinnen bzw. Schüler die durch ihre Lehrkräfte mit sonderpädagogischem Förderbedarf ausgewiesen wurden, gehen nicht mit in die Analyse ein. 
Tab. 2 Kennwerte der verwendeten Indikatoren (Mittelwerte ungewichtet)

\begin{tabular}{|c|c|c|c|c|c|c|c|}
\hline & Min & $\begin{array}{l}\text { 1. Quar- } \\
\text { til }\end{array}$ & Median & Mittelw & $\begin{array}{l}\text { 3. Quar- } \\
\text { til }\end{array}$ & Max & $\mathrm{SD}$ \\
\hline Leseverstehensleistung & $-1,97$ & 0,02 & 0,38 & 0,39 & 0,79 & 2,33 & 0,61 \\
\hline $\begin{array}{l}\text { Streuung der Leseverste- } \\
\text { hensleistung }\end{array}$ & 0,46 & 1,17 & 1,30 & 1,30 & 1,43 & 2,51 & 0,22 \\
\hline $\begin{array}{l}\text { Teilnahmequote Leseverste- } \\
\text { hen }\end{array}$ & 0,47 & 0,91 & 0,95 & 0,94 & 1 & 1 & 0,07 \\
\hline Mathematikleistung & $-2,01$ & $-0,45$ & $-0,12$ & $-0,11$ & 0,23 & 1,88 & 0,52 \\
\hline $\begin{array}{l}\text { Streuung der Mathematik- } \\
\text { leistung }\end{array}$ & 0 & 1,39 & 1,57 & 1,58 & 1,77 & 2,98 & 0,32 \\
\hline Teilnahmequote Mathematik & 0,4 & 0,92 & 0,95 & 0,94 & 1 & 1 & 0,07 \\
\hline $\begin{array}{l}\text { Anteil der Schülerinnen und } \\
\text { Schüler mit Lernmittelfrei- } \\
\text { heit }\end{array}$ & 0 & 14,03 & 20,00 & 22,52 & 28,52 & 73,53 & 12,40 \\
\hline $\begin{array}{l}\text { Anteil der Schülerinnen und } \\
\text { Schüler mit nichtdeutscher } \\
\text { Familiensprache }\end{array}$ & 0 & 4,62 & 13,55 & 19,11 & 29,24 & 93,91 & 18,21 \\
\hline $\begin{array}{l}\text { Anteil der Schülerinnen und } \\
\text { Schüler mit sonderpädagogi- } \\
\text { schem Förderbedarf }\end{array}$ & 0 & 0 & 0 & 1,102 & 0 & 16,45 & 2,59 \\
\hline $\begin{array}{l}\text { Schulen mit Ganztagsange- } \\
\text { boten }\end{array}$ & 0 & 0 & 0 & 0,35 & 1 & 1 & 0,48 \\
\hline $\begin{array}{l}\text { Wöchentlich erteilte Unter- } \\
\text { richtsstunden je } 100 \text { Schü- } \\
\text { ler*innen }\end{array}$ & 106 & 136,5 & 150 & 152,7 & 163,6 & 281,8 & 22,94 \\
\hline Schüler*innen je Klasse & 9,50 & 15,75 & 17,59 & 17,60 & 19,50 & 24,94 & 2,44 \\
\hline $\begin{array}{l}\text { Jahrgangsübergreifende } \\
\text { Klassen }\end{array}$ & 0 & 0 & 0 & 0,12 & 0 & 1 & 0,32 \\
\hline $\begin{array}{l}\text { Von nicht voll qualifizier- } \\
\text { ten Lehrkräften erteilter } \\
\text { Wochenstundenanteil }\end{array}$ & 0 & 0 & 3,20 & 3,85 & 5,70 & 36,30 & 4,04 \\
\hline
\end{tabular}

\subsection{Verwendeter Datensatz}

Im zweiten Arbeitsschritt wurden diese Leistungsinformationen mit den aus den Schulstatistiken gewonnen Daten zusammengeführt und alle Fälle eliminiert, für die eines der beiden Datenpakete fehlte. Der so gewonnene Datensatz umfasst Informationen zu 872 öffentlichen Grundschulen in Rheinland-Pfalz ${ }^{7}$. Tab. 2 enthält deskriptive Daten zu den im Weiteren genutzten Variablen ${ }^{8}$.

In Tab. 3 sind die Zusammenhänge zwischen den VERA3-Ergebnissen, den Merkmalen der Schulzusammensetzung und den schulorganisatorischen Merkma-

\footnotetext{
${ }^{7} \mathrm{Da}$ in allen Schulen mit sehr wenigen Schülerinnen und Schülern in der dritten Jahrgangsstufe mindestens 2/3 (Mittelwert: 98\%) tatsächlich auch an der VERA-Testung teilgenommen haben, stellen die ermittelten Schulwerte trotz kleiner Fallzahlen relativ zuverlässige Schätzungen dar und wurden daher nicht aus dem Datensatz entfernt.

${ }^{8}$ Die Variable „Schülerzahl je Klasse“ ist normalverteilt, die weiteren metrischen Variablen sind mehr oder weniger linkssteil, weil niedrige Werte unterhalb des arithmetischen Mittelwerts überwiegen. Die Variablen zur Ganztagsschule und jahrgangsübergreifenden Klassen sind dichotom (ja/nein).
} 
len dargestellt. Die Kompetenzen der Schülerinnen und Schüler werden über den Leistungsmittelwert der Schülerinnen und Schüler (M) und über die Streubreite (SD) der Schülerleistungen bei den VERA3-Erhebungen erfasst. Zusätzlich wird der Anteil der Schülerinnen und Schüler berücksichtigt, der an den Schulen an den Leistungserhebungen teilgenommen hat (TN-quote). Zwischen den Schülerleistungen und der durchschnittlichen sozialen Lage der Schülerinnen und Schüler einer Schule (Anteil der Schülerinnen und Schüler mit Lernmittelfreiheit bzw. nichtdeutscher Familiensprache) gibt es die erwarteten hohen negativen Korrelationen. Als Folge der durchschnittlich niedrigeren sozialen Lage der Schülerinnen und Schüler der Schulen mit einem hohen Anteil von Inklusionsschülerinnen und -schülern fallen dort die Leistungsergebnisse eher ungünstig aus. Auffällig sind die größeren schulinternen Leistungsstreuungen an Schulen mit hohem Anteil von Inklusionsschülern, obwohl diese Schülerinnen und Schüler selbst (insgesamt 696) bei den Analysen nicht berücksichtigt wurden. Schließlich sind die Zusammenhänge zwischen sozialer Situation der Schülerinnen und Schüler an den Schulen und der Teilnahmequote an den VERA-Erhebungen beachtenswert, weil mit abnehmender durchschnittlicher sozialer Lage der Schülerinnen und Schüler die Teilnahmequote an den Schulen sinkt.

Bemerkenswert ist außerdem der nicht nennenswerte Zusammenhang zwischen den erteilten Unterrichtsstunden je Schüler und den Schülerleistungen. Während demnach die personelle Ausstattung der Schulen keinen Zusammenhang mit den durchschnittlichen Schülerleistungen an den Schulen zeigt, bestehen sie zwischen durchschnittlicher Schülerleistung und den schulorganisatorischen Bedingungen. In größeren Klassen sind die Schülerleistungen tendenziell schlechter, in Schulen mit jahrgangsübergreifenden Klassen eher besser. Der negative Zusammenhang zwischen einem Ganztagsangebot und den durchschnittlichen Schülerleistungen lässt sich darauf zurückführen, dass Ganztagsangebote eher an Schulen mit einer durchschnittlich niedrigeren sozialen Lage der Schülerinnen und Schüler existieren (s. Tab. 1).

\section{Ergebnisse}

Bezogen auf die erste Fragestellung verdeutlichen die Korrelationskoeffizienten (Tab. 3), dass mit dem Anteil der Schülerinnen und Schüler mit nichtdeutscher Familiensprache die Vorhersage des Leseverstehens (Varianzaufklärung 16\%) besser gelingt als für Mathematik (Varianzaufklärung knapp 11\%). Noch besser gelingt die Vorhersage der VERA3-Ergebnisse mit dem Anteil der Schülerinnen und Schüler mit Lernmittelbefreiung. Auch hier ist die Vorhersage der Leseverstehensleistung (Varianzaufklärung 23\%) besser als die Vorhersage der Mathematikleistung (Varianzaufklärung $20 \%$ ).

Die Addition der beiden Prädiktoren (s. Tab. 4) zeigt, dass die beiden Prädiktoren zu einem erheblichen Anteil die gleiche Varianz aufklären. Der Gewinn an zusätzlich aufgeklärter Varianz beträgt für das Leseverstehen 1,4\%, für Mathematik hingegen nur etwa $0,3 \%$. Der Vergleich der Beta-Koeffizienten weist aus, dass die Lernmittelbefreiung die prädiktionsmächtigere Variable ist, wobei die Reduktion des Beta- 
Tab. 3 Zusammenhänge (Korrelationskoeffizienten) zwischen Merkmalen der Zusammensetzung der Schülerschaft, schulorganisatorischen Bedingungen an den Grundschulen und den bei VERA3 erfassten Leistungen in Deutsch und Mathematik in Rheinland-Pfalz 2015/16

\begin{tabular}{|c|c|c|c|c|c|c|}
\hline \multirow{2}{*}{$\begin{array}{l}\text { Erfasste Merkmale der } \\
\text { Schülerleistung } \\
\text { Merkmale der Zusammenset- } \\
\text { zung der Schulen und schulor- } \\
\text { ganisatorische Merkmale }\end{array}$} & \multicolumn{3}{|c|}{ Leseverstehensleistung } & \multicolumn{3}{|c|}{ Mathematikleistung } \\
\hline & M & SD & $\begin{array}{l}\text { TN- } \\
\text { quote }\end{array}$ & $\mathrm{M}$ & SD & $\begin{array}{l}\text { TN- } \\
\text { quote }\end{array}$ \\
\hline Anteil mit Lernmittelfreiheit & $-0,469 * *$ & 0,052 & $-0,288^{* *}$ & $-0,436 * *$ & 0,049 & $-0,261 * *$ \\
\hline $\begin{array}{l}\text { Anteil mit nichtdeutscher } \\
\text { Familiensprache }\end{array}$ & $-0,391 * *$ & 0,061 & $-0,184 * *$ & $-0,320 * *$ & 0,053 & $-0,187 * *$ \\
\hline $\begin{array}{l}\text { Anteil mit sonderpädagogi- } \\
\text { schem Förderbedarf }\end{array}$ & $-0,108^{* *}$ & $0,079^{*}$ & $-0,229 * *$ & $-0,110 * *$ & $0,095^{* *}$ & $-0,187^{* *}$ \\
\hline $\begin{array}{l}\text { Schulen mit Ganztagsangebo- } \\
\text { ten }\end{array}$ & $-0,183^{* *}$ & $0,109^{* *}$ & $-0,148^{* *}$ & $-0,207 * *$ & $0,070^{*}$ & $-0,159 * *$ \\
\hline $\begin{array}{l}\text { Wöchentlich erteilte Unter- } \\
\text { richtsstunden je } 100 \text { Schü- } \\
\text { ler*innen }\end{array}$ & $-0,016$ & 0,038 & $-0,090 * *$ & $-0,006$ & $-0,022$ & $-0,058$ \\
\hline Schüler*innen je Klasse & $-0,144 * *$ & $0,067 *$ & $-0,136^{* *}$ & $-0,173 * *$ & $0,078^{*}$ & $-0,131^{* *}$ \\
\hline $\begin{array}{l}\text { Jahrgangsübergreifende Klas- } \\
\text { sen }\end{array}$ & $0,084 *$ & $-0,031$ & $0,101 * *$ & $0,096^{* *}$ & $-0,066$ & $0,067^{*}$ \\
\hline $\begin{array}{l}\text { Von nicht voll qualifizierten } \\
\text { Lehrkräften erteilter Wochen- } \\
\text { stundenanteil }\end{array}$ & $-0,076^{*}$ & $0,068^{*}$ & $-0,053$ & $-0,056$ & $-0,024$ & $-0,054$ \\
\hline
\end{tabular}

$* p<0,05 ; * * p<0,01$

Tab. 4 Multiple Regression der Schulleistungsmittelwerte auf die Prädiktoren der sozialen Situation der Schülerinnen und Schüler

\begin{tabular}{lllll}
\hline Kriteriumsvariable & \multicolumn{2}{l}{ Leseverstehensleistung } & \multicolumn{2}{l}{ Mathematikleistung } \\
\hline Prädiktoren & Beta & $p$ & Beta & $p$ \\
$\begin{array}{l}\text { 1. Anteil mit nichtdeutscher Familien- } \\
\text { sprache }\end{array}$ & $-0,159$ & 0,000 & $-0,067$ & 0,093 \\
2. Anteil mit Lernmittelfreiheit & $-0,371$ & 0,000 & $-0,393$ & 0,000 \\
- & $\mathrm{R}^{2}$ & Adj. $\mathrm{R}^{2}$ & $\mathrm{R}^{2}$ & Adj. $\mathrm{R}^{2}$ \\
Varianzaufklärung & $23,39 \%$ & $23,21 \%$ & $19,28 \%$ & $19,10 \%$ \\
\hline
\end{tabular}

Koeffizienten (gegenüber den bivariaten Zusammenhängen; s. Tab. 3) für die Mathematikleistung geringer ausfällt als für die Leseverstehensleistung. In Analysen unter Einbezug des Anteils von Schülerinnen und Schülern mit sonderpädagogischem Förderbedarf, erweist sich diese Variable als nicht weitergehend signifikant prädiktiv für die Schülerleistung (hier nicht weiter dargestellt). Im Rückbezug auf die Fragestellung 1 zeigt sich also, dass sowohl der Anteil mit nichtdeutscher Familiensprache als auch der Anteil mit Lernmittelfreiheit auch in der gemeinsamen Betrachtung in engem Zusammenhang mit der Leistung stehen, es also zu einem additiven Zusammenwirken kommt. Demgegenüber erklärt der Anteil von Schülerinnen und Schülern mit sonderpädagogischem Förderbedarf bei gemeinsamer Betrachtung keine weiteren Anteile der Leistungsunterschiede. 
Tab. 5 Multiple Regression der Schulleistungsmittelwerte auf die Prädiktoren der sozialen Situation der Schülerinnen und Schüler unter Einbezug eines multiplikativ verknüpften Interaktionsterms

\begin{tabular}{|c|c|c|c|c|}
\hline \multirow{2}{*}{$\begin{array}{l}\text { Kriteriumsvariable } \\
\text { Prädiktoren }\end{array}$} & \multicolumn{2}{|c|}{ Leseverstehensleistung } & \multicolumn{2}{|c|}{ Mathematikleistung } \\
\hline & Beta & $P$ & Beta & $p$ \\
\hline $\begin{array}{l}\text { 1. Anteil mit nichtdeutscher Familien- } \\
\text { sprache }\end{array}$ & $-0,086$ & 0,221 & 0,002 & 0,980 \\
\hline 2. Anteil mit Lernmittelfreiheit & $-0,328$ & 0,000 & $-0,349$ & 0,000 \\
\hline 3. Interaktionsterm $(1 . * 2$.) & $-0,002$ & 0,224 & $-0,002$ & 0,247 \\
\hline - & $\mathrm{R}^{2}$ & Adj. $\mathrm{R}^{2}$ & $\mathrm{R}^{2}$ & Adj. $\mathrm{R}^{2}$ \\
\hline Varianzaufklärung & $23,51 \%$ & $23,25 \%$ & $19,41 \%$ & $19,13 \%$ \\
\hline
\end{tabular}

Mit Blick auf den letzten Aspekt der ersten Forschungsfrage (verstärkende Effekte bei kombiniertem Auftreten) wurde darüber hinaus auch die Interaktion der beiden signifikanten Prädiktoren betrachtet, operationalisiert als Produkt der beiden Variablen. Dieser multiplikative Interaktionsterm trägt zur Vorhersage der Leistungsergebnisse in beiden Leistungsdomänen nicht beachtenswert bei (s. Tab. 5). Es lässt sich folglich über das additive Zusammenwirken hinaus keine weitere Verstärkung der Leistungsunterschiede zwischen den Schulen feststellen, wenn beide hier berücksichtigten sozialen Merkmale der Schülerinnen und Schüler zusammenfallen. Die Forschungsfrage 1 kann also wie folgt beantwortet werden: Es gibt erhebliche Unterschiede zwischen Schulen, die auf additiv wirksame Effekte der Anteile von Schülerinnen und Schülern mit Befreiung von der Lernmittelbezahlung (stärkerer Effekt) und mit nichtdeutscher Familiensprache (schwächerer Effekt) zurückzuführen sind, während der Anteil von Schülerinnen und Schülern mit sonderpädagogischem Förderbedarf keinen weiteren Einfluss auf die Leistungsunterschiede besitzt.

Um zu verdeutlichen, welche praktischen Implikationen diese Zusammenhänge haben, sind in Abb. 1 die mittleren Leseverstehens- und Mathematikleistungen als Boxplots dargestellt. Damit kann zugleich die zweite Ausgangsfrage beantwortet werden. Dabei wird für die beiden zentralen Variablen der Schulzusammensetzung (Anteil der Lehrmittelbefreiung, Anteil der Schülerinnen und Schüler mit nichtdeutscher Familiensprache) eine Aufteilung der Schulen vorgenommen, indem das am ungünstigsten sozial zusammengesetzte Viertel der Schulen (oberstes Viertel) den mittleren $50 \%$ der Schulen und den sozial günstig zusammengesetzten $25 \%$ der Schulen (unterstes Viertel) gegenübergestellt werden.

Es wird deutlich, dass die Leistungsverteilungen (bei vergleichbar großen Streuungen) erheblich verschoben ausfallen. Für den Anteil der Lernmittelbefreiung liegen in beiden Leistungsbereichen die Grenzen des 3. Quartils unterhalb des Medianwertes der mittleren $50 \%$ der Schulen, für die Gruppierung nach dem Anteil der nichtdeutschen Familiensprache liegen sie nur marginal darüber. Zugleich liegen die Medianwerte der Schulen mit ungünstigen sozialen Bedingungen jeweils unter der ersten Quartilsgrenze der Mittelgruppe von Schulen. Im Vergleich der sozial günstig zusammengesetzten Schulen mit den mittleren $50 \%$ zeigt sich, dass sich die Verteilungen in Mathematik kaum unterscheiden während im Leseverstehen erwartungsgemäße Unterschiede erkennbar werden. Diese fallen jedoch erheblich geringer aus, als die Unterschiede zwischen der Mittelgruppe und den Schulen mit ungünstigen sozialen Bedingungen. Dies gilt sowohl für die Gruppierung hinsicht- 

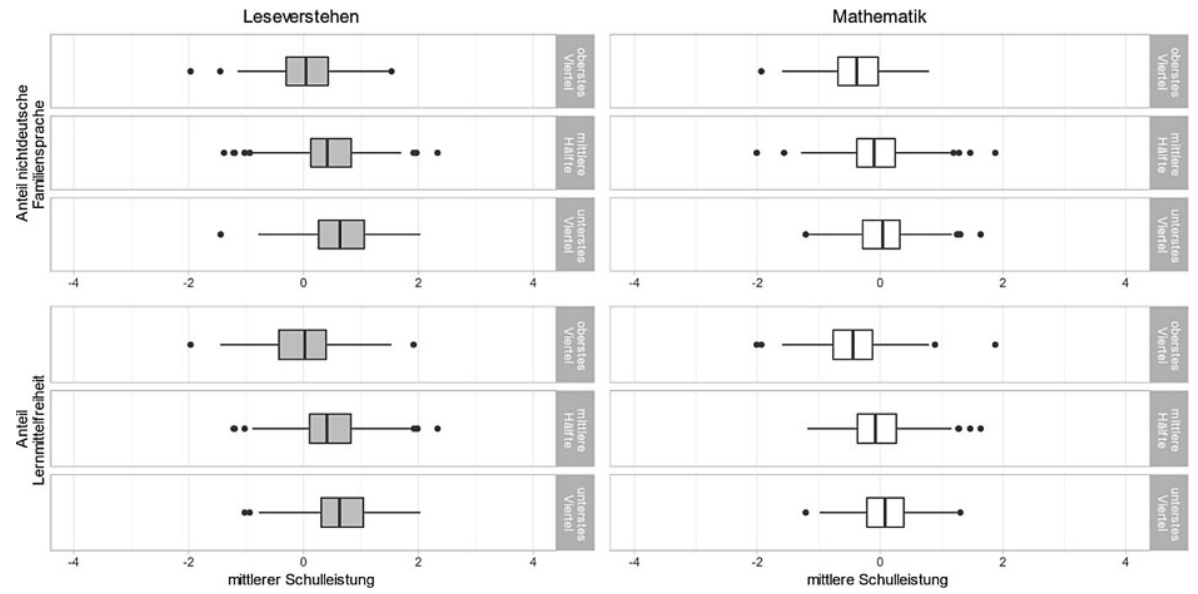

Abb. 1 Gegenüberstellung mittleren Leistungen der jeweils sozial ungünstig zusammengesetzten Schulen (oberes Quartil) mit den restlichen 75\% der Schulen (Boxplot, dicke Linie: Median, Ränder der Box: 1. bzw. 3. Quartil)

lich des Anteils der nichtdeutschen Familiensprache als auch für die Gruppierung bezüglich des Anteils der Lernmittelbefreiung. Betrachtet man die Mittelwerte der beiden Extremgruppen, dann ergeben sich für die Gruppierung nach dem Anteil der Schülerinnen und Schüler mit nichtdeutscher Familiensprache Unterschiede, die etwa dem Lernfortschritt von mehr als 1,5 Schuljahren im Leseverstehen und fast einem Schuljahr in Mathematik entsprechen ${ }^{9}$. Noch dramatischer fällt der Effekt für die Gruppierung nach dem Anteil der Lernmittelbefreiung aus: Im Leseverstehen entspricht die Differenz nicht ganz 1,75, in Mathematik nicht ganz 1,25 Schuljahren. Es zeigt sich also hinsichtlich der zweiten Forschungsfrage nach der Größe der Leistungsunterschiede, dass die betrachteten Merkmale einen größeren Effekt auf die Leseverstehensleistungen als auf die Mathematikleistungen besitzen und dass diese Unterschiede erheblich sind. Knapp ein Viertel (23\%) der Leseverstehensleistungsunterschiede zwischen rheinland-pfälzischen Grundschulen sind durch die Anteile der Lernmittelbefreiung und nicht-deutscher Familiensprache determiniert. Für Mathematik ist das Ausmaß mit knapp einem Fünftel (19\%) etwas geringer, aber immer noch bedeutend. Die Betrachtung der Extremgruppen zeigt, dass es hier um differentielle Lernentwicklungen geht, die mehr als ein Schuljahr ausmachen können.

Zur Beantwortung der dritten Frage wurde ein (exploratives) Pfadmodell gerechnet, in das die schulorganisatorischen Merkmale als vermittelnde Prozessvariablen des Zusammenhangs zwischen Schülermerkmalen und den gemessenen Schülerleis-

\footnotetext{
9 Hierzu werden die Leistungswerte auf die Metrik der Bildungsstandards umgerechnet und die Differenzen dieser Werte werden anschließend ins Verhältnis zu den aus Querschnittsanalysen geschätzten Lernzuwächsen der jeweiligen Domänen gesetzt (vgl. Wittig und Weirich 2017; Haag und Kohrt 2017).

$10 \mathrm{Da}$ es sich hier um ein exploratives Vorgehen handelt, wurden jeweils alle möglichen Einflusspfade modelliert, es handelt sich also um saturierte Modelle. Dementsprechend lassen sich keine Aussagen zur Modellpassung treffen, etwa auf der Grundlage des RMSEA oder anderer Fit-Indizes.
} 


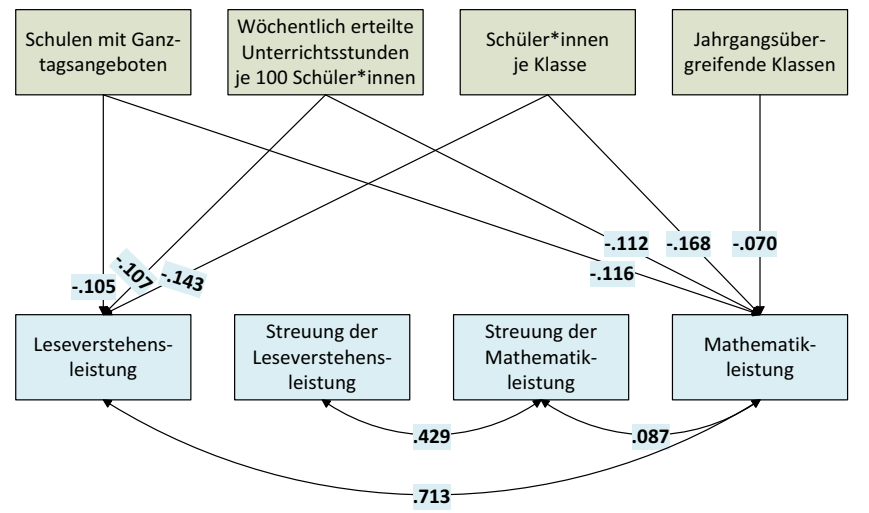

Merkmale der Schulorganisation

Abb. 2 Vorhersagen der Leistungsvariablen mit Hilfe der schulorganisatorischen Merkmale (standardisierte Koeffizienten mit $p<0,05)$

tungen aufgenommen werden ${ }^{10}$. Als Kriteriumsvariablen dienten jeweils der Schulmittelwert sowie die schulinterne Standardabweichung der in VERA gemessenen Leseverstehens- bzw. Mathematikleistungen. Dabei bauen wir das Modell in drei Schritten auf, in dem zunächst jeweils nur zwei der drei Merkmalsgruppen analysiert werden, bevor das Gesamtmodell dargestellt wird. Hier unberücksichtigt bleiben die Teilnahmequoten an der VERA-Erhebung, weil keine Effekte auf die Schülerleistungen (weder auf die Mittelwerte noch auf die Streuungen) beobachtet werden konnten und die Zusammenhänge mit den Schülermerkmalen unterschiedliche Interpretationen zulassen (z. B. unterschiedlicher Krankenstand nach Sozialgruppen). Ebenfalls nicht berücksichtigt wird der Anteil des von nicht voll qualifizierten Lehrkräften erteilten Unterrichts, trotz des leichten Zusammenhangs mit den Schülerleistungen, weil dies ein Zusammenhang ist, der weder mit sonstigen Merkmalen der Schulorganisation und insbesondere nicht den Schülermerkmalen variiert.

In einem ersten Modell wurde analysiert, inwiefern die schulorganisatorischen Merkmale die Leistungsvariablen prädizieren können (s. Abb. 2).

Es zeigt sich, dass alle Pfadkoeffizienten negative Vorzeichen besitzen und es nur in Bezug auf die Leistungsmittelwerte signifikante Pfade gibt. Während die Leseverstehensleistung durch den Status als Ganztagsschule, die Anzahl der wöchentlich je 100 Schülerinnern und Schülern erteilten Unterricht und die Klassengröße signifikant prädiziert werden kann (Anteil erklärter Varianz 4,7\%), sagen alle vier betrachteten Merkmale der Schulorganisation die mittlere Mathematikleistung signifikant vorher (Anteil erklärter Varianz: 6,1\%). Für die schulinternen Streuungsmaße ergeben sich keine signifikanten Prädiktoren, der Anteil der aufgeklärten Varianz liegt bei $1,7 \%$ (Lesen) bzw. 1,0\% (Mathematik) Prozent. Während ein negativer Einfluss der Klassengröße auf die Leistung nicht überrascht, erscheint ein negativer Einfluss der Unterrichtsquantität kontraintuitiv und auch negative Effekte der Ganztagsbeschulung erscheinen bemerkenswert. Es liegt nahe, dass diese Effekte mit Unterschieden in der Zusammensetzung der Schülerschaften einhergehen. 


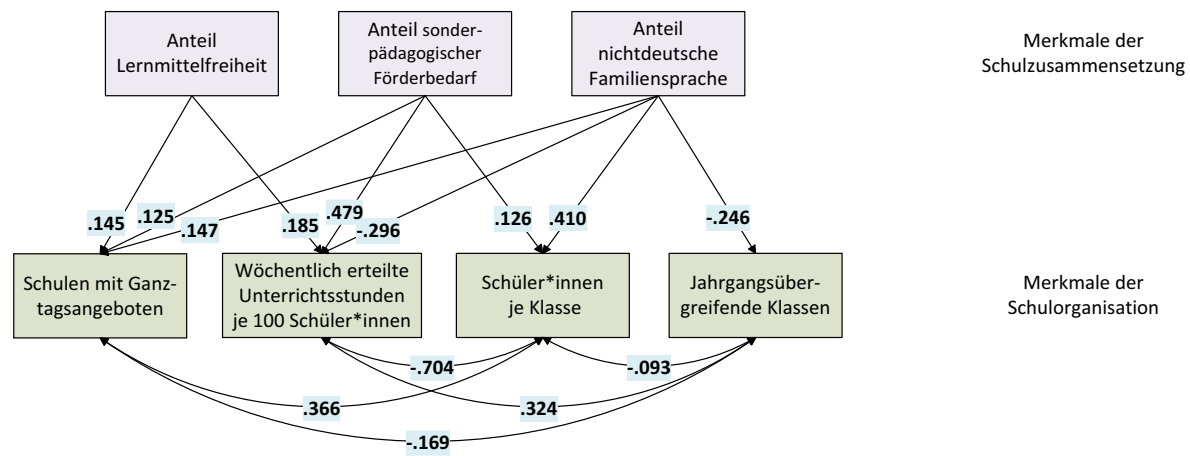

Abb. 3 Vorhersagen der schulorganisatorischen Merkmale mit Hilfe der Merkmale der Schulzusammensetzung (standardisierte Koeffizienten mit $p<0,05$ )

Im zweiten Schritt wurden daher die Beziehungen zwischen den Merkmalen der Schulzusammensetzung und den Merkmalen der Schulorganisation untersucht (s. Abb. 3).

Es zeigt sich, dass der Anteil von Schülerinnen und Schülern mit nichtdeutscher Familiensprache alle vier Variablen der Schulorganisation vorhersagt. Dabei gehen hohe Anteile sehr deutlich mit größeren Klassen und in geringem Umfang mit dem Status als Ganztagsschule sowie einer in mittlerem Ausmaß niedrigeren Wochenstundenzahl erteilten Unterrichts und dem Fehlen von jahrgangsübergreifenden Klassen einher. Der Anteil von Schülerinnen und Schülern mit sonderpädagogischem Förderbedarf steht sehr deutlich mit der Unterrichtsquantität und schwach sowohl mit größeren Klassen als auch mit dem Status als Ganztagsschule in positivem Zusammenhang. Der Anteil der Schülerinnen und Schüler mit Lernmittelbefreiung prädiziert in schwachem Ausmaß den Ganztagsschulstatus und die Anzahl der erteilten Wochenstunden. Insgesamt zeigt sich, dass die erklärten Varianzanteile in diesem Modell im allgemeinen höher ausfallen als in Modell 1: Während der Aufklärungsanteil für die Jahrgangübergreifenden Klassen mit 5,8\% ähnlich niedrig ausfällt wie für die Leistungsmittelwerte, liegen die Anteile für die Ganztagsschule $(10,0 \%)$, die Klassengröße $(18,3 \%)$ und die erteilten Wochenstunden $(27,4 \%)$ erheblich darüber.

Insgesamt enthält das Muster der Beziehungen einerseits sehr plausible Zusammenhänge, wie den stärksten hier erkennbaren zwischen sonderpädagogischem Förderbedarf und Unterrichtszeit, der primär dem Einsatz zusätzlicher Förderlehrkräfte geschuldet sein dürfte. Andererseits erscheint zum einen bemerkenswert, dass der Ganztagsschulstatus nur mäßig von den Merkmalen der (niedrigen) sozialen Zusammensetzung der Schulen determiniert wird und dass der Anteil nichtdeutscher Familiensprache vergleichsweise eng mit der Klassengröße assoziiert ist. Es kann angenommen werden, dass dies ein Effekt städtischer Ballungsgebiete mit mehrzügigen Grundschulen sein könnte.

Im dritten Schritt wurde nun die Beziehung zwischen allen drei Merkmalsebenen untersucht (siehe Abb. 4). Es ist ersichtlich, dass die Vorhersagen der Merkmale der Schulzusammensetzung auf die Merkmale der Schulorganisationsebene (Mo- 


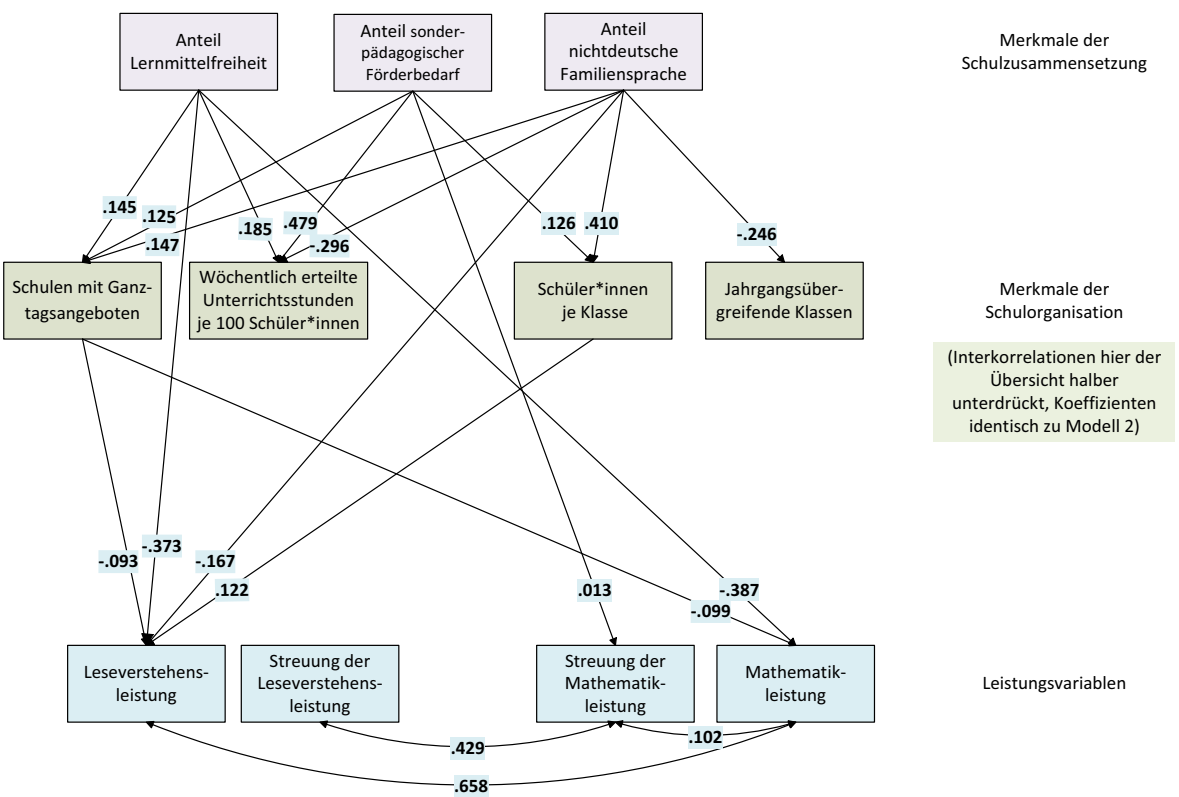

Abb. 4 Vorhersagen der Leistungsvariablen mit Hilfe der schulorganisatorischen Merkmale in Kombination mit den Vorhersagen durch die Merkmale der Schulzusammensetzung (standardisierte Koeffizienten mit $p<0,05)$

dell 2, s. Abb. 3) unverändert bleiben. Darüber hinaus sagen alle drei Merkmale der Schulzusammensetzung jeweils mindestens eine der Leistungsvariablen signifikant vorher. Während sich der Anteil der Lernmittelfreiheit stark negativ auf die Mittelwerte beider Leistungsdomänen auswirkt, prädiziert der Anteil der nichtdeutschen Familiensprache die Leseverstehensleistung deutlich schwächer, aber ebenfalls mit negativem Vorzeichen. Der Anteil der Schülerinnen und Schüler mit sonderpädagogischem Förderbedarf prädiziert sehr schwach (aber auf dem 5\%-Fehlerniveau signifikant) höhere schulinterne Streuungen der Mathematikleistungen.

Mit Blick auf die Pfade zwischen den Merkmalen der Schulorganisation und den Leistungsvariablen ergeben sich gegenüber der isolierten Betrachtung im ersten Modell (s. Abb. 2) deutliche Veränderungen. Die Effekte der Ganztagsschulangebote bleiben weiterhin signifikant und reduzieren sich nur geringfügig. Der zuvor dargestellte negative Pfad der Unterrichtsquantität auf die Leistungsmittelwerte wechselt in beiden Domänen das Vorzeichen und verfehlt mit $p=0,057$ für das Leseverstehen die Signifikanzgrenze nur knapp. Der ohnehin schwache Pfad zwischen Jahrgangsübergreifenden Klassen und der Mathematikleistung reduziert sich und verfehlt die Signifikanzgrenze nun deutlich. Während sich ohne Berücksichtigung der Schulzusammensetzung signifikante negative Effekte der Klassengröße auf beide Leistungsmittelwerte ergaben, weisen nun beide Koeffizienten positive Vorzeichen auf, wobei der Pfad auf die Mathematikleistung klar nicht signifikant ausfällt.

Die Anteile der aufgeklärten Varianz liegen in diesem Modell bei 24,1\% für die mittlere Leseverstehensleistung und bei 20,2\% für die mittlere Mathematikleistung. 
Hinsichtlich der dritten Forschungsfrage nach den vermittelnden Effekten schulorganisatorischer Variablen und der Personalausstattung lässt sich also festhalten, dass nur kleine Effekte für Ganztagsangebote (negativ) und die Klassengröße (positiv, nur für Leseverstehen) bestehen. Der Indikator für die Personalausstattung (wöchentlich erteilte Unterrichtsstunden je 100 Schüler*innen) weist einen knapp nicht signifikanten positiven Zusammenhang mit der Leseverstehensleistung auf. Insgesamt lassen sich durch die Berücksichtigung der schulorganisatorischen Bedingungen bzw. der Personalausstattung jeweils nur weniger als $1 \%$ der Leistungsunterschiede in beiden Domänen zusätzlich erklären.

\section{Diskussion}

Die Analysen bestätigen den bekannten engen negativen Zusammenhang zwischen den durchschnittlichen Leistungen der Schülerinnen und Schüler und der sozialen Zusammensetzung der Schülerschaft einer Schule. Da neben den Leistungen der einzelnen Schülerinnen und Schüler keine sozialen Individualmerkmale vorliegen, können über die Auswirkungen der sozialen Zusammensetzung der Schülerinnen und Schüler auf die individuelle Leistung keine Aussagen getroffen werden. Konsistent mit dem bisherigen Forschungsstand ist ebenfalls die größere Bedeutung der sozialen Lage im Vergleich zur Migrationssituation. Vor allem die häufiger bestehenden sprachlichen Defizite bei den Schülerinnen und Schülern mit Migrationshintergrund dürften die Ursache für den Zusammenhang zwischen nichtdeutscher Familiensprache und der Leseverstehensleistung sein. Bei der Mathematikleistung verschwindet dieser Zusammenhang. Die Leistungsunterschiede zwischen dem durch die soziale Zusammensetzung der Schülerinnen und Schüler besonders benachteiligten Viertel von Schulen und den übrigen Schulen sind ganz erheblich, denn bei beiden erfassten Merkmalen der sozialen Lage und beiden Leistungsfächern liegen die Durchschnittleistungen an den Schulen mit niedrigen sozialen Ausgangsbedingungen unter den Leistungen des unteren Leistungsviertels bei den übrigen Schulen. Die Leistungen an den Schulen mit günstigen Bedingungen der sozialen Zusammensetzung der Schülerinnen und Schüler heben sich nochmals - wenn auch nicht so deutlich - von den durchschnittlichen Schulen ab.

An den Grundschulen mit einer durchschnittlich niedrigen sozialen Lage der Schülerinnen und Schüler sind die Klassenfrequenzen durchschnittlich deutlich höher und die Zahl der erteilten Unterrichtsstunden je Schüler eher niedriger als an den Schulen mit einer günstigeren sozialen Zusammensetzung der Schülerinnen und Schüler. Erwartet wurde, dass diese ungünstigen schulorganisatorischen und personellen Bedingungen sich für den Lernprozess der Schülerinnen und Schüler neben der sozialen Lage auf die Schülerleistungen auswirken. Diese Erwartung konnten die Datenanalysen aber nicht erfüllen. Im Blick auf die internationale Schulforschung ist dies Ergebnis allerdings nicht überraschend. Die Klassengröße hat als solche keinen direkten Einfluss auf die Leistung, sondern nur einen über Merkmale der Instruktion vermittelten. Dass sich dann solche Effekte hier nicht nachweisen lassen mag zum Einen an den Schwierigkeiten von Lehrkräften liegen, ihren Unterricht an unterschiedliche Klassengrößen für Schüler wahrnehmbar zu adaptieren zum ande- 
ren aber auch an der Tatsache, dass damit Effekte auf der Klassenebene beschrieben werden, die sich in diesen Daten durch die Aggregation auf Schulebene nivellieren. Untersuchungen zur hierarchischen Struktur von Schulleistungsvarianzen zeigen im Regelfall, dass der Ebene der Klasse eine erheblich größere Bedeutung zukommt, als der Ebene der Schulen (s. Hosenfeld et al. 2001).

Ähnlich ist anzunehmen, dass auch die wöchentlich erteilten Unterrichtsstunden je Schülerin und Schüler zwischen den Schulen in sehr unterschiedlicher Weise über den Pflichtunterricht hinaus verwendet werden (z. B. für Arbeitsgemeinschaften, Projekte) und Instruktionszeit nicht mit aktiver Lernzeit gleichzusetzen ist. Insofern verweisen die Befunde auf das zentrale Ergebnis der Hattie-Studie, dass es für den Lernerfolg der Schülerinnen und Schüler auf die Lehrkräfte ankommt (Lotz und Lipowsky 2015). Zu vermuten ist allerdings ein durchschnittlich erhöhter pädagogischer Einsatz der Lehrkräfte an den Schulen mit ungünstigen sozialen Ausgangsbedingungen, damit die dort zusätzlich durchschnittlich ungünstigeren schulorganisatorischen und personellen Bedingungen nicht doch die Leistungsunterschiede zwischen den Schulen noch verstärken.

Bezogen auf das Ganztagsangebot lassen sich die Zusammenhänge dahingehend interpretieren, dass ein offenes Ganztagsangebot in der in Rheinland-Pfalz im Schuljahr 2015/2016 realisierten Form an Schulen mit einer eher sozial ungünstig zusammengesetzten Schülerschaft nicht dazu beiträgt soziale Benachteiligungen zu verringern.

Die empirische Schulforschung konzentrierte sich bisher vor allem auf Merkmale des Unterrichts, um den Lehrkräften Hinweise zu geben, wie sie ihren Unterricht verbessern und die Lernentwicklung aller Schülerinnen und Schüler positiv beeinflussen können. Weniger im Blick ist die Frage, welche Maßnahmen der Schulpolitik und -administration geeignet sind, die Rahmenbedingungen für die Lehrkräfte bei ihren Bemühungen zu verbessern, damit sie noch erfolgreicher arbeiten können. Aus den Ergebnissen dieser Studie den Schluss zu ziehen, dass schulpolitisches und -administratives Handeln für die Lernentwicklung der Schülerinnen und Schüler wenig bedeutsam ist, erscheint uns aber voreilig, weil beispielsweise Merkmale der Lehrkräfte (tatsächliche Arbeitszeit, Fluktuation in den Kollegien, Krankenstand etc.) nicht berücksichtigt wurden. Vielmehr sollte der Frage verstärkt nachgegangen werden, welche Rahmenbedingungen Lehrkräfte benötigen, damit sie effektiv arbeiten können.

Funding Open Access funding enabled and organized by Projekt DEAL.

Open Access Dieser Artikel wird unter der Creative Commons Namensnennung 4.0 International Lizenz veröffentlicht, welche die Nutzung, Vervielfältigung, Bearbeitung, Verbreitung und Wiedergabe in jeglichem Medium und Format erlaubt, sofern Sie den/die ursprünglichen Autor(en) und die Quelle ordnungsgemäß nennen, einen Link zur Creative Commons Lizenz beifügen und angeben, ob Änderungen vorgenommen wurden.

Die in diesem Artikel enthaltenen Bilder und sonstiges Drittmaterial unterliegen ebenfalls der genannten Creative Commons Lizenz, sofern sich aus der Abbildungslegende nichts anderes ergibt. Sofern das betreffende Material nicht unter der genannten Creative Commons Lizenz steht und die betreffende Handlung nicht nach gesetzlichen Vorschriften erlaubt ist, ist für die oben aufgeführten Weiterverwendungen des Materials die Einwilligung des jeweiligen Rechteinhabers einzuholen. 
Weitere Details zur Lizenz entnehmen Sie bitte der Lizenzinformation auf http://creativecommons.org/ licenses/by/4.0/deed.de.

\section{Literatur}

Autorengruppe Bildungsberichterstattung (2016). Bildung in Deutschland 2016. Ein indikatorengestützter Bericht mit einer Analyse zu Bildung und Migration. Bielefeld: wbv.

Avenarius, H., Ditton, H., Döbert, H., Klemm, K., Klieme, E., Rürup, M., Tenorth, H.-E., Weishaupt, H., \& Weiß, M. (2003). Bildungsbericht für Deutschland. Erste Befunde. Opladen: Leske + Budrich.

Bayerisches Landesamt für Schule. Abteilung Qualitätsagentur (2018). Bildungsbericht Bayern 2018. Gunzenhausen: ISB. https://www.isb.bayern.de/download/21663/bildungsbericht_bayern_2018_ barrierefrei.pdf. Zugegriffen: 04.12.2020.

Beierle, S., Hoch, C., \& Reißig, B. (2019). Schulen in benachteiligten sozialen Lagen. Untersuchung zum aktuellen Forschungsstand mit Praxisbeispielen. Halle: DJI. https://www.dji.de/fileadmin/ user_upload/bibs2019/28019_DJI_Schulen_in_benachteiligten_sozialen_Lagen.pdf. Zugegriffen: 09.03.2020.

Bremm, N. (2020). Umso mehr kommt es auf die Lehrperson an - Defizitperspektiven von Lehrkräften an Schulen in sozialräumlich benachteiligten Lagen. In S. Druck \& D. Bruland (Hrsg.), Kritische Lebensereignisse und Herausforderungen für die Schule (S. 107-128). Weinheim: Beltz Juventa.

Bremm, N., Klein, E.D., \& Racherbäumer, K. (2016). Schulen in „schwieriger“ Lage ?! Begriffe, Forschungsbefunde und Perspektiven. Die Deutsche Schule, 108, 323-339.

Ditton, H., \& Krüsken, J. (2006). Sozialer Kontext und schulische Leistungen - zur Bildungsrelevanz segregierter Armut. Zeitschrift für Soziologie der Erziehung und Sozialisation, 26, 135-157.

Dumont, H., Neumann, M., Maaz, K., \& Trautwein, U. (2013). Die Zusammensetzung der Schülerschaft als Einflussfaktor für Schulleistungen: Internationale und nationale Befunde. Psychologie in Erziehung und Unterricht, 60, 163-183.

Finke, G., \& Lange, S. (2012). Segregation an Grundschulen: Der Einfluss der elterlichen Schulwahl. Sachverständigenrat deutscher Stiftungen für Integration und Migration (SVR) - Forschungsbereich. Berlin: SVR. https://www.svr-migration.de/wp-content/uploads/2014/11/Segregation_an_ Grundschulen_SVR-FB_WEB.pdf. Zugegriffen: 27.02.2020.

Haag, N., \& Kohrt, P. (2017). Mittelwerte und Streuungen der im Fach Mathematik erreichten Kompetenzen. In P. Stanat, S. Schipolowski, C. Rjosk, S. Weirich \& N. Haag (Hrsg.), IQB-Bildungstrend 2016. Kompetenzen in den Fächern Deutsch und Mathematik am Ende der 4. Jahrgangsstufe im zweiten Ländervergleich (S. 168-186). Münster: Waxmann. https://www.iqb.hu-berlin.de/bt/ BT2016/Bericht/BT2016_Bericht.pdf. Zugegriffen: 03.10.2020.

Hanschmann, F., \& Weishaupt, H. (2017). Schulstatistik: Zu Schwierigkeiten des Datenzugangs und der Datenbereitstellung im Bundesstaat. SchulVerwaltung, 22(6), 164-166. Ausgabe Hessen und Rheinland-Pfalz.

Helbig, M., \& Jähnen, S. (2018). Wie brüchig ist die soziale Architektur unserer Städte? Trends und Analysen der Segregation in 74 deutschen Städten. Discussion Paper P-2018-001. Berlin: WZB. https:// bibliothek.wzb.eu/pdf/2018/p18-001.pdf. Zugegriffen: 13.08.2020

Helbig, M., \& Nikolai, R. (2019). Bekommen die sozial benachteiligsten Schüler*innen die „besten “ Schulen? Eine explorative Studie über den Zusammenhang von Schulqualität und sozialer Zusammensetzung von Schulen am Beispiel von Berlin. Discussion Paper P-2019-002. Berlin: WZB.

Hosenfeld, I., Helmke, A., Ridder, A., \& Schrader, F.-W. (2001). Eine mehrebenenanalytische Betrachtung von Schul- und Klasseneffekten. Empirische Pädagogik, 15, 513-534.

Jeworutzki, S., Knüttel, K., Niemand, C., Schmidt, B.-J., Schräpler, J.-P., \& Terpoorten, T. (2017). Räumlich segregierte Bildungsteilhabe in NRW und im Ruhrgebiet. In J.-P. Schräpler, S. Jeworutzki, B. Butzin, T. Terpoorten, J. Goebel \& G.G. Wagner (Hrsg.), Wege zur Metropole Ruhr. ZEFIR Materialien, (Bd. 6, S. 13-213). Bochum: Zentrum für interdisziplinäre Regionalforschung (ZEFIR).

Kemper, T. (2017). Die schulstatistische Erfassung des Migrationshintergrundes in Deutschland. Journal für Educational Research online, 9, 144-168.

Klemm, K., Hoffmann, L., Maaz, K., \& Stanat, P. (2018). Privatschulen in Deutschland. Trends und Leistungsvergleiche. Berlin: Friedrich-Ebert-Stiftung.

Laschke, C. (2010). Schülerleistungen und regionaler Kontext - Eine Zusammenhangsanalyse für Berliner Grundschulen. Zeitschrift für amtliche Statistik Berlin-Brandenburg, 4, 77-88. https://www.statistikberlin-brandenburg.de/Produkte/Zeitschrift/2010/HZS_1006.pdf. Zugegriffen: 26.09.2020. 
Lotz, M., \& Lipowsky, F. (2015). Die Hattie-Studie und ihre Bedeutung für den Unterricht - Ein Blick auf ausgewählte Aspekte der Lehrer-Schüler-Interaktion. In G. Mehlhorn, F. Schulz \& K. Schöppe (Hrsg.), Begabungen entwickeln \& Kreativität fördern (S. 97-136). München: kopaed. https://www.uni-kassel.de/fb01/fileadmin/datas/fb01/Institut_fuer_Erziehungswissenschaft/ Dateien/Grundschulpaedagogik/Pr\%C3\%BCfung/Lotz_Lipowsky_2015-Die_Hattie-Studie_und_ ihre_Bedeutung_f\%C3\%BCr_den_Unterricht_01.pdf. Zugegriffen: 26.09.2020.

Morris-Lange, S., Wendt, H., \& Wohlfarth, C. (2013). Segregation an deutschen Schulen. Ausmaß, Folgen und Handlungsempfehlungen für bessere Bildungschancen. Berlin: Sachverständigenrat der deutschen Stiftungen für Integration und Migration (SVR) - Forschungsbereich. https://www.stiftungmercator.de/media/downloads/3_Publikationen/SVR_Studie_Bildungssegregation_Juli_2013.pdf. Zugegriffen: 09.03.2020.

Mundelius, M. (2019). Der Kerndatensatz auf der Basis von Individualdatenerhebungen in der Schulstatistik. Von Summendaten zu Einzeldaten. In D. Fickermann \& H. Weishaupt (Hrsg.), Bildungsforschung mit Daten der amtlichen Statistik. Die Deutsche Schule 14. Beiheft. (S. 38-45).

Richter, D., Marx, A., \& Zorn, D. (2018). Lehrkräfte im Quereinstieg: sozial ungleich verteilt? Eine Analyse zum Lehrermangel an Berliner Grundschulen. Gütersloh: Bertelsmann Stiftung.

Rjosk, C., Haag, N., Heppt, B., \& Stanat, P. (2017). Zuwanderungsbezogene Disparitäten. In P. Stanat, S. Schipolowski, C. Rjosk, S. Weirich \& N. Haag (Hrsg.), IQB-Bildungstrend 2016. Kompetenzen in den Fächern Deutsch und Mathematik am Ende der 4. Jahrgangsstufe im zweiten Ländervergleich (S. 237-275). Münster: Waxmann. https://www.iqb.hu-berlin.de/bt/BT2016/Bericht/BT2016_ Bericht.pdf. Zugegriffen: 03.10.2020.

Schuchart, C., Schneider, K., Weishaupt, H., \& Riedel, A. (2012). Welchen Einfluss hat die Wohnumgebung auf die Grundschulwahl von Eltern? Analysen zur Bedeutung von kontextuellen und familiären Merkmalen. Empirische Pädagogik, 26, 504-534.

Sekretariat der ständigen Konferenz der Kultusminister der Länder in der Bundesrepublik Deutschland (KMK) (2020). Schüler, Klassen, Lehrer und Absolventen der Schulen 2009 bis 2018. (Statistische Veröffentlichungen der Kultusministerkonferenz. Dokumentation Nr. 224 - März 2020). https:// www.kmk.org/fileadmin/Dateien/pdf/Statistik/Dokumentationen/Dok224_SKL2018.pdf. Zugegriffen: 29.09.2020.

Ständige Konferenz der Kultusminister der Länder in der Bundesrepublik Deutschland (2006). Gesamtstrategie der Kultusministerkonferenz zum Bildungsmonitoring. Neuwied: Luchterhand.

Ständige Konferenz der Kultusminister der Länder in der Bundesrepublik Deutschland (2016). Gesamtstrategie der Kultusministerkonferenz zum Bildungsmonitoring. Berlin: Wolters Kluwer. https://www. kmk.org/fileadmin/Dateien/veroeffentlichungen_beschluesse/2015/2015_06_11-GesamtstrategieBildungsmonitoring.pdf. Zugegriffen: 12.06.2018

Sundsboe, A. (2015). Warum benachteiligt schulische Segregation die „Bildungsfernen“? In L. Hertel, T. Fölker \& N. Pfaff (Hrsg.), Brennpunkt (-)Schule. Zum Verhältnis von Schule, Bildung und urbaner Segregation (S. 49-66). Opladen: Budrich.

Weishaupt, H. (2016). Schulen in schwieriger Lage und Schulfinanzierung. Die Deutsche Schule, 108, 354-369.

Weishaupt, H. (2018a). Kinderarmut und Migration: Personalausstattung der Schulen und schulische Lernbedingungen. HLZ, 71, 24-25. Ausgabe Hessen.

Weishaupt, H. (2018b). Regionale Unterschiede der Bildungsausstattung als Ursache für Bildungsbenachteiligung - das Beispiel Rheinland-Pfalz. In M. Sixt, M. Bayer \& D. Müller (Hrsg.), Bildungsentscheidungen und lokales Angebot: Die Bedeutung der Infrastruktur für Bildungsentscheidungen im Lebenslauf (S. 247-273). Münster: Waxmann.

Weishaupt, H. (2020). Sonderpädagogische Förderung im Schuljahr 2016/17 (Teil 2). SchulVerwaltung, 25, 12-15. Ausgabe Hessen/Rheinland-Pfalz.

Weishaupt, H., \& Kemper, T. (2016). Stellenzuweisung über einen Sozialindex: Alternativen zu den bislang in Nordrhein-Westfalen verwendeten Indikatoren. SchulVerwaltung, 27, 341-343. Ausgabe Nordrhein-Westfalen.

Wittig, J., \& Weirich, S. (2017). Mittelwerte und Streuungen der im Fach Deutsch erreichten Kompetenzen. In P. Stanat, S. Schipolowski, C. Rjosk, S. Weirich \& N. Haag (Hrsg.), IQB-Bildungstrend 2016. Kompetenzen in den Fächern Deutsch und Mathematik am Ende der 4. Jahrgangsstufe im zweiten Ländervergleich (S. 153-167). Münster: Waxmann. https://www.iqb.hu-berlin.de/bt/BT2016/ Bericht/BT2016_Bericht.pdf. Zugegriffen: 03.10.2020.

Ziegler, C., \& Richter, D. (2017). Der Einfluss fachfremden Unterrichts auf die Schülerleistung: Können Unterschiede in der Klassenzusammensetzung zur Erklärung beitragen? Unterrichtswissenschaft, 45, 136-155. 